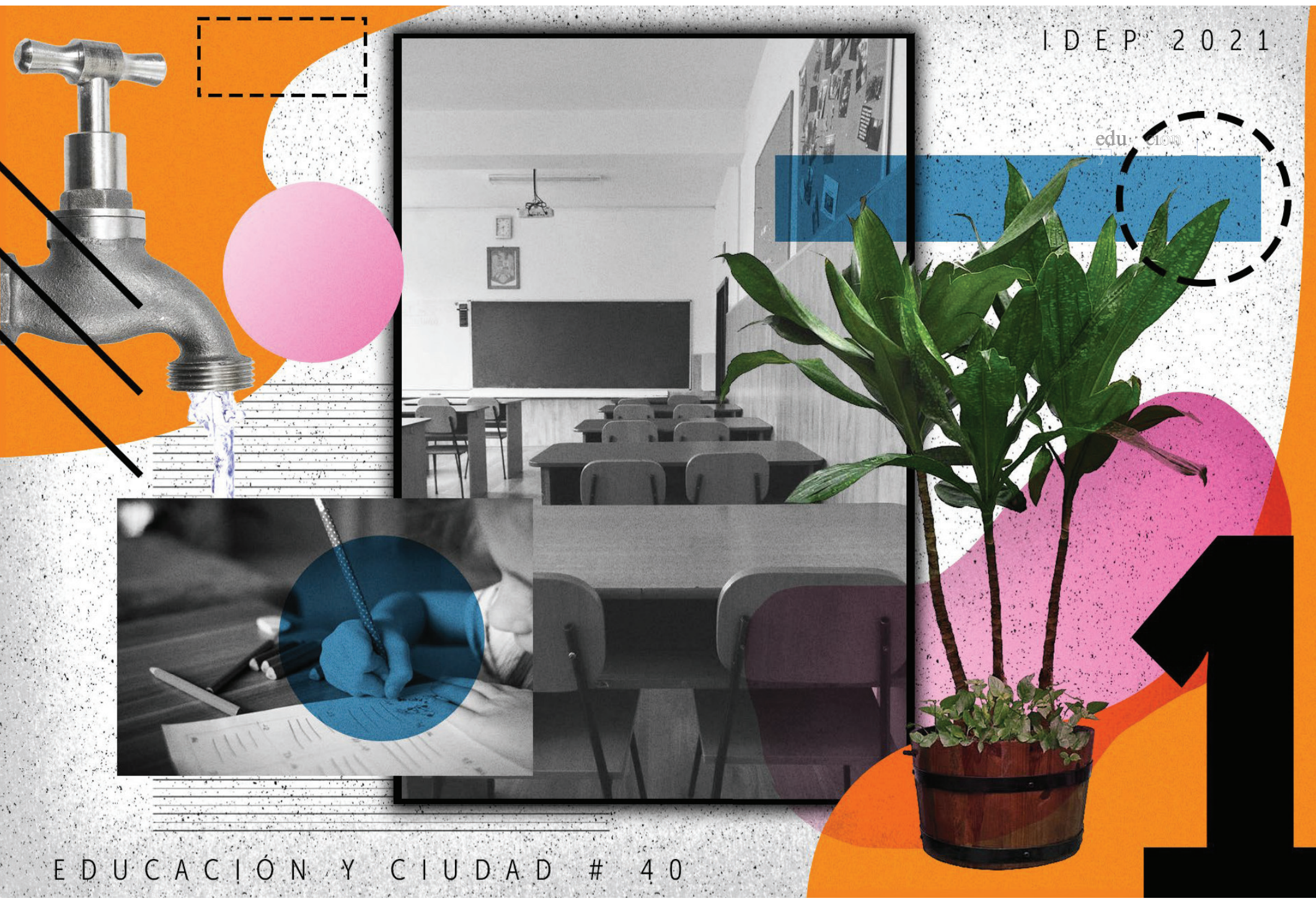

\title{
Incursión del discurso ambiental en la escuela de Colombia
}

INCURSION OF ENVIRONMENTAL DISCOURSE IN SCHOOLS IN COLOMBIA INCURSÃO DO DISCURSO AMBIENTAL NAS ESCOLAS DA COLÔMBIA Juan José Zapata Franco 
Juan José Zapata Franco ${ }^{1}$

1. Magíster en Educación, Universidad Pedagógica Nacional de Colombia, línea de Educación, Pedagogía y Subjetividades; ORCID: https://orcid.org/00000001-9661-2447; correo electrónico: jjzapataf@upn.edu.co

\section{Citar artículo como:}

Zapata, J. (2021, Enero-Junio). Incursión del discurso ambiental en la escuela de Colombia. Revista Educación y Ciudad, No. 40, pp. 17-33. //doi.org/10.36737/01230425.n40.2020.2454

DOI: https://doi.org/10.36737/01230425.n40.2020.2454

Fecha de recepción: 3 de agosto de 2020 / Fecha de aprobación: 4 de noviembre de 2020

\section{Resumen}

La presente investigación se realizó siguiendo el método arqueológico-genealógico propuesto por Michel Foucault. Así, se construyó el archivo del discurso ambiental que circuló a finales del Siglo XX, a partir de una extensa búsqueda en fuentes primarias que incluyó periódicos, libros escolares, lineamientos curriculares y legislación; lo cual permitió develar que la emergencia del discurso ambiental en la escuela, bajo el nombre de Educación Ambiental, se dio desde la tensión entre diversas fuerzas de tipo económico, político y social, ejercidas por instancias gubernamentales, educativas, internacionales y la sociedad civil.

Palabras clave: Educación ambiental, historia, arqueología, genealogía, legislación.

\begin{abstract}
The present investigation was carried out following the archaeological-genealogical method proposed by Michel Foucault. Thus built the archive of the environmental discourse that circulated at the end of the 20th century, based on an extensive search in sources elementary schools that included newspapers, school books, curricular guidelines and legislation; which allowed to reveal that the the emergence of the environmental discourse at school, under the name of Environmental Education, arose from the tension between various economic, political and social forces, exercised by governmental, educational, international bodies and civil society.
\end{abstract}

Keywords: Environmental education, history, archeology, genealogy, legislation.

\section{Resumo}

A presente investigação foi realizada seguindo o método arqueológico-genealógico proposto por Michel Foucault. então construiu o arquivo do discurso ambientalista que circulou no final do século XX, a partir de uma extensa busca em fontes escolas primárias que incluíam jornais, livros escolares, diretrizes curriculares e legislação; o que permitiu revelar que o a emergência do discurso ambiental na escola, com o nome de Educação Ambiental, surgiu da tensão entre várias forças econômicas, políticas e sociais, exercidas por organizações governamentais, educacionais e internacionais e a sociedade civil.

Palavras-chave: Educação ambiental, história, arqueologia, genealogia, legislação. 


\section{La problemática se vuelve evidente}

T uego de la Segunda Guerra Mundial, la presión Lsobre el medio ambiente se incrementó de manera abrupta; por un lado, el crecimiento poblacional desembocó en un "Boom" en las naciones comunistas y Estados Unidos, país que contaba con una sociedad de clase media exigente en materia de productos de consumo y una producción a escala industrial que debía responder a tales demandas, lo cual llevó a los dos bloques a combatir en una multitud de frentes alrededor del mundo para lograr los materiales que les permitieran funcionar. Resultado de esta guerra, la carrera armamentista e industrial se enfocó en el uso de energía nuclear, cuya producción dio paso a un tipo de residuos con un grado de peligro y contaminación nunca antes vistos; estas, entre otras razones, generaron gran preocupación por el estado de los ecosistemas y la disponibilidad, a futuro, de recursos naturales.

A finales de los años sesenta, el escenario político y social se veía conmocionado debido a una multitud de manifestaciones impulsadas por movimientos, principalmente estudiantiles, con reivindicaciones sociales de todo tipo, que respondían al surgimiento de una conciencia global y una sociedad más informada sobre los acontecimientos del mundo, gracias a las nuevas formas de comunicación y a un mayor acceso a la educación superior. Publicaciones como La bomba demográfica de Paul Ehrlich, y La primavera silenciosa, de Rachel Carson, llamaron la atención sobre las afectaciones de las actividades en la naturaleza; para el caso de Carson, se destaca el uso de pesticidas sintéticos como causa de la desaparición de ciertas especies y la contaminación de ecosistemas que anteriormente eran prístinos.

Este tipo de publicaciones aumentó el interés y las preocupaciones del público general por el medio ambiente, especialmente en los países desarrollados, que comenzaban a ver su calidad de vida afectada por las actividades industriales, lo cual impulsó la creación de movimientos ecologistas que se sumaron a las reclamaciones alrededor del mundo en el '68, con reivindicaciones por los Derechos Humanos, laborales, feministas y animalistas, y posiciones contra el capitalismo y la sociedad de consumo.

La problemática alcanzó instancias internacionales por incidentes como el desastre de Minamata en Japón, cuando una fábrica de fertilizantes causó el envenenamiento de la bahía por vertimiento de mercurio, ocasionando la muerte de cientos de personas, afectando la salud de miles y contaminando todo un ecosistema; durante más de diez años el hecho fue encubierto hasta que una revuelta de pescadores le dio visibilidad internacional, forzando al gobierno japonés a emprender una investigación. Al tiempo, se destacaba la situación de los veteranos de la guerra de Vietnam, quienes presentaban desordenes físicos y mentales al regresar del frente, vinculados al uso que Estados Unidos hizo de defoliantes químicos, como el Agente Naranja, para destruir al enemigo, arrasando selvas y campos y causando graves afectaciones a la salud de la población y los combatientes (Black, 2012).

Durante la misma década, en Colombia también se dieron movilizaciones, especialmente estudiantiles y campesinas, que reclamaban mayor participación 
política, acceso a servicios públicos, satisfacción de las necesidades materiales de la población y redistribución de tierras. A pesar de que dichas manifestaciones aún responden a una visión utilitarista de la naturaleza, viéndola como mercancía con valor comercial cuyo propósito es el crecimiento económico de la nación, la clase media, intelectual y académica, la clase obrera y trabajadora, y los campesinos, presentan un reconocimiento de la problemática ambiental, ligado a las reivindicaciones sociales y la defensa de los recursos naturales. Ante su presión, el gobierno del Frente Nacional creó el Inderena que, junto a las Corporaciones Regionales, la declaración de Parques Nacionales y la creación del Jardín Botánico, marcó el comienzo de la institucionalización del ambientalismo en el país.

La década de los setenta se caracterizó por un cambio en la idea de que la sociedad occidental implicaba necesariamente progreso, pues, impulsada por las crisis del petróleo, nucleares y económicas, abandonó los ideales de cambios sociales radicales para lograr una sociedad más justa, intensificando, desde los medios de comunicación, las campañas a favor del consumismo y promoviendo una idea individualista de bienestar, presionando así, de maneras inéditas, la producción y explotación de recursos, como materias primas y combustibles, llevando a que los organismos internacionales se preguntaran por la idea de un crecimiento infinito en un planeta finito.

En tal contexto se produce el informe Los límites del crecimiento (Meadows, Meadows, Randers, y Behrens, 1972), presentado para la Cumbre Mundial de Estocolmo en 1972, proponiendo una epistemología ecológica que considera la interconexión de todos los hechos, los cuales, por más pequeños que sean, tendrán una repercusión planetaria; esta idea, expuesta en la cumbre, responde a las preocupaciones ambientales por el aumento en la acidez de los lagos suecos, causada por la lluvia ácida proveniente de las centrales termoeléctricas inglesas, arrastradas por la corriente del Golfo (Regodón, 2009). Frente a ello se introduce el eslogan "Piensa global, actúa local", que será base del enfoque pedagógico activo, el cual busca promover las acciones locales y comunitarias para la solución de problemas ambientales dentro de la EA en las posteriores conferencias sobre el tema (Bastidas, 2013).

Por su parte, las preocupaciones ambientales comienzan a manifestarse en el discurso colombiano a principios del Siglo XX, debido principalmente a la afectación económica causada por la explotación despreocupada de los recursos naturales que, para ese momento, era práctica común en el país. Posteriormente, también se vieron impulsadas por agentes externos, conferencias y publicaciones científicas y literarias de países desarrollados, donde comenzaban a ser evidentes los efectos adversos de la industrialización en el ambiente y la calidad de vida. Debido a que el país basaba su economía en la explotación agrícola, empezaron a trascender preocupaciones frente a la degradación y caída de la productividad de los suelos, provocada por la erosión y el uso de pesticidas sintéticos.

\section{Ambientalismo en Colombia}

El Inderena, encabezado por Margarita Marino de Botero, promueve en 1985 la "Campaña verde"; con la distribución de cartillas, y el lema "Mil alcaldes siembran futuro", buscó crear, con ayuda de las autoridades municipales de todo el país, y 
en conjunto con la población local, los "Concejos verdes”, los cuales tenían el objetivo combinado de proteger el medio ambiente y promover la iniciativa comunal, la democracia y el poder ciudadano, con la convicción de que "el desarrollo y el progreso deben descansar sobre un manejo y cuidado adecuado del medio ambiente como patrimonio común" (Inderena, 1985).

A finales de 1985 se habían creado 700 concejos verdes en todo el país, con objetivos específicos de conservación que incluían la realización de un inventario del patrimonio público natural, el cual se debía actualizar anualmente para llevar un control del estado de conservación de bosques y cuencas; al tiempo, se concretó la creación de un vivero de especies nativas y se adelantaron campañas de reforestación en zonas afectadas, con la participación de la población local.

Durante el proceso se dio importancia a la participación de los jóvenes en las campañas ecológicas, fomentando la inclusión de temas ambientales en las escuelas y colegios mediante el uso de las cartillas, que fueron repartidas a los representantes del sector educativo en los concejos verdes, y la promoción de concursos periódicos entre los jóvenes; igualmente, se organizaron jornadas de recolección de plantas y semillas con los estudiantes de educación primaria, y a los pertenecientes a la educación media se les encargó la tarea de pensar, junto a la comunidad, en soluciones frente a problemas inmediatos relacionados con el medio ambiente (Inderena, 1985).

Al momento de liquidación del Inderena, y de creación del Ministerio del Medio Ambiente, se habían publicado 800.000 cartillas con fines educativos para el área ambiental no formal en $E l$ Tiempo y El Espectador, al igual que las llamadas EcoCartillas del medio ambiente, dirigidas a maestros, que funcionaban como guías metodológicas para la labor educativa:

Su contenido es por consiguiente, en primer lugar, una recopilación de las actividades y objetivos propuestos al inicio de cada unidad y, en segundo término, una serie de recomendaciones y reflexiones pedagógico-didácticas sobre el mantenimiento y defensa del medio ambiente (Vargas, 1993).

Los temas tratados no solo consideraban los ambientes urbanos, incluyendo apartados dirigidos a la recolección, disposición y tratamiento de basuras, la contaminación producida por vehículos o el ruido; sino también aspectos propios del medio rural, como el control de la erosión a través de programas de reforestación, la defensa de la vegetación natural, el control de inundaciones, el desarrollo de prácticas adecuadas de cultivo o el control de la aplicación de agroquímicos (Inderena, 1985).

El fortalecimiento en la opinión pública del activismo y de la nueva conciencia ambiental, logra que el tema sea uno de los más relevantes durante la Asamblea Constituyente de 1991, particularmente en las discusiones de la Comisión Quinta, enfocada en temas económicos, sociales y ecológicos. En total, se realizaron dos ponencias directamente vinculadas al tema y se presentaron tres proyectos de ley que buscaron atender las necesidades del país al respecto, destacándose el propuesto por el representante liberal Guillermo Perry Rubio, que consagraba el deber del Estado de garantizar el derecho colectivo a un ambiente sano. 
Junto a ello, también se propuso la inclusión, en la Carta Magna, del concepto de desarrollo sostenible para el manejo de recursos naturales, y la garantía de la calidad de vida. Al tiempo, junto a los cuatro artículos ambientales específicos, otros 26 artículos hacen referencia directa al tema ambiental, entre ellos, frente a nociones como: función social y ecológica de la propiedad privada; posibilidad de declarar emergencias ecológicas; obligación de la Contraloría de incluir en la contabilidad nacional el valor de los recursos naturales; y la inclusión de la EA en la educación formal y no formal; entre otros que llevaron a conocer la nueva Constitución como "La carta verde" (Sánchez, 1991).

\section{Legislación ambiental en Colombia}

En el ámbito jurídico nacional, los primeros índices que apuntan a la formulación de una política de conservación ambiental responden a la necesidad de preservar el recurso hídrico de uso para la agricultura, por medio de la reglamentación de la tala de bosques presentes en las cuencas hídricas. Así, la Ley 119 de 1919, especifica dentro del código fiscal las medidas sobre la explotación y delimitación de los bosques nacionales: "Artículo Primero: Se consideran como bosques nacionales las plantaciones naturales de caucho, tagua, pita, henoquéa, quina, balata, jengibre, maderas preciosas y demás productos de exportación o de consumo interior, existentes en terrenos de la Nación". Dicha Ley crea, a su vez, la Comisión Forestal, encargada de la vigilancia de bosques nacionales, baldíos y aguas de uso público, creada, para este momento, con un fin más estadístico, pues no existía un inventario claro de los recursos que se explotaban, su cantidad y si el trabajo se realizaba en terrenos de la Nación.

Junto a ello, una de las primeras medidas que buscó la protección del patrimonio nacional fue la declaración de las pendientes occidentales de la Sierra Nevada de Santa Marta como Reserva Forestal Nacional, por medio del Decreto 178 de 1933. El objetivo de esta clasificación era conservar las fuentes de agua que proveían a las vastas plantaciones bananeras en la base de la montaña. Todas las actividades agrícolas, incluyendo el pastoreo, fueron prohibidas en los límites de la reserva, con excepción de las plantaciones de café o, si fuese el caso, por medio de algún estudio científico que probara la no afectación de la corriente en volumen o calidad. Dichas medidas se oficializan con el Decreto 1383 de 1940, que consolida las Zonas Forestales Protectoras; así, según el Artículo Primero:

Artículo Primero: Se determina Zona Forestal Protectora al conjunto de terrenos que, por su topografía, o por su ubicación en las cabeceras de las cuencas hidrográficas y márgenes de depósitos o cursos permanentes de agua, conviene que permanezcan revestidos de masas arbóreas por la acción que éstas ejercen sobre el régimen fluvial, conservación de aguas y suelos, salubridad de los centros urbanos, etc.

Tal planteamiento responde a la necesidad de garantizar el suministro de agua en una economía predominantemente agraria, específicamente cuando, por ejemplo, se deben proveer las exigentes plantaciones de caña de azúcar en el Valle del Cauca; lo cual explica la creación de las primeras reservas en las zonas comprometidas de la cuenca del Río Cauca, donde se presentaba el desarrollo agroindustrial 
azucarero. Todo fue soportado institucionalmente con la creación en 1954, por parte del Ministerio de Economía, de la Corporación Autónoma Regional del Valle del Cauca, que sería la primera de su tipo en el país, encargada de la administración de los recursos naturales de la cuenca, así como de la realización de proyectos que lleven a su mejor aprovechamiento (Lenis, 2013).

En tal contexto, y pese a que Colombia no ratificó la Convención Panamericana para la protección de la flora, de la fauna, y de las bellezas escénicas naturales de los países de América, de acuerdo con el Ministro de Relaciones Exteriores de la época, el delegado oficial, Dr. Gabriel Turbay, apoyó las recomendaciones generales, impulsando así la creación de normas conservacionistas en el país. De acuerdo con ello, al siguiente año se implementó la primera regulación activa en contra de la cacería, buscando proteger ciertas especies de aves mediante el establecimiento de temporadas de cacería y de una moratoria en la cacería de aves por diez años (Megank, 1975).

Por su parte, la primera designación de Reserva Biológica y Estación en Campo fue creada con el Decreto 52 de 1948, por el Presidente Mariano Ospina Pérez, en la Sierra de la Macarena, designando el comienzo de una serie de medidas oficiales para la conservación natural que, aunque al principio se dieron con un financiamiento restringido y acciones de corto alcance, marcaron el comienzo de un lento proceso de institucionalización. Debieron pasar seis años para que tuviese lugar el siguiente avance sustantivo en materia de conservación: para febrero 3 de 1954, el Ministro de Agricultura, Brigadier General Arturo Charry, teniendo en cuenta las recomendaciones de la Unión Internacional para la
Conservación de la Naturaleza (UICN), declaró al Cóndor Andino como el ave nacional de Colombia, convirtiéndose de esta forma en la primera especie completamente protegida del país, al igual que en otros países de Latinoamérica (Megank, 1975).

Durante la siguiente década se observó la creación de las correspondientes corporaciones autónomas en las regiones, determinadas por las cuencas relevantes para la conservación, desde su valor en cuanto a capacidad para proveer el recurso hídrico, o su contenido de maderas valiosas para la explotación forestal, aspectos que fueron luego comprendidos por el Artículo 13 de la Ley 2 de 1959, donde específicamente se afirma:

El Gobierno puede reservar para destinar como Parque Nacional Natural los sitios o terrenos que por sus bellezas naturales, riqueza de su fauna o su flora, particularidades geológicas o hidrológicas deben ser objeto de medidas especiales de protección.

Lo cual se emprendió siguiendo el modelo de protección empleado por Estados Unidos para instituir, en 1872, el Parque Yellowstone como reserva natural, a pesar de que causó el desplazamiento de varias comunidades indígenas, desconociendo el factor humano presente en la región que, además, hace parte de las interacciones del ecosistema. Luego, en 1960, se declaró la Cueva de los Guácharos como el primer Parque Nacional Natural del país, después de que la UNESCO la nombrara "Reserva de la Biósfera", por ser hogar de esta especie endémica de pájaros (Barra, 2010), retirando la zona del parque de la jurisdicción de baldíos nacionales y asignándola a la administración de la Corporación para los Valles del Sinú y el Magdalena, que manejó los parques nacionales y fue base de la creación, en 
1968, del Instituto Nacional de Recursos Naturales Renovables (Inderena) (Lenis, 2013).

\section{La Educación Ambiental en Colombia}

El Código Nacional de Recursos Naturales Renovables y Protección al Medio Ambiente, promulgado en el Decreto 2911 de 1974 (República de Colombia, 1974), se presenta como el primer documento oficial que menciona el "ambiente", entendiéndole como el espacio en que habitan e interactúan todos los elementos vivos y no vivos presentes en el ecosistema, incluyendo a los seres humanos, y determinando que ellos dependen del mismo para su supervivencia y el desarrollo económico, por lo que deben asumir su responsabilidad frente a su cuidado; el Artículo Primero dicta:

El ambiente es patrimonio común. El Estado y los particulares deben participar en su preservación y manejo, que son de utilidad pública e interés social. La preservación y manejo de los recursos naturales renovables también son de utilidad pública e interés social (República de Colombia, Decreto 2911 de 1974).

El Código sigue las recomendaciones de la primera Cumbre de la Tierra, realizada en Estocolmo durante 1972, contando con la participación de una delegación nacional. Allí, partiendo de la relación entre medio ambiente y necesidades de desarrollo en los países, se declara el derecho de disfrutar de un ambiente sano para el pleno desarrollo intelectual, moral, social y espiritual; de igual manera, se reconoce la dependencia frente al medio ambiente para el sustento material, adjudicando a las instituciones locales la potestad de formular normas y medidas al respecto, mientras se insta a la cooperación internacional para tratar los problemas cada vez más evidentes a escala mundial.

Tanto el Código, como las declaraciones de la cumbre, expresan que conseguir los objetivos propuestos implica necesariamente lograr que la población, joven y adulta, especialmente en los sectores menos favorecidos, cuente con un constante ejercicio educativo alrededor del tema, para:

[...] ensanchar las bases de una opinión pública bien informada, y de una conducta de los individuos, de las empresas y de las colectividades inspirada en el sentido de su responsabilidad en cuanto a la protección y mejoramiento del medio ambiente en toda su dimensión humana (Naciones Unidas, 1972).

Como respuesta, en el Artículo 14 del Código Nacional de Recursos Naturales se solicita al gobierno nacional una reglamentación acorde para la educación primaria, secundaria y universitaria, de tal manera que fuese posible:

a) Incluir cursos sobre ecología, preservación ambiental y recursos naturales renovables.

b) Fomentar el desarrollo de estudios interdisciplinarios.

c) Promover la realización de jornadas ambientales con participación de la comunidad, y de campañas de educación popular, en los medios urbanos y rurales, para lograr la comprensión de los problemas del ambiente, dentro del ámbito en el cual se presentan (República de Colombia, Decreto 2911 de 1974).

Así, se da el comienzo a la formulación de una serie de estrategias enfocadas a instaurar la Educación Ambiental (E.A.) en la educación formal colombia- 
na, tales como la creación en 1978 de la Comisión Asesora para la Educación Ecológica y Ambiental, encargada de formalizar la inclusión de cursos sobre ecología, preservación ambiental y recursos naturales renovables, dentro de un modelo interdisciplinar, en los currículos del sector formal.

La comisión está en concordancia con las propuestas de la Carta de Belgrado, producto del primer Seminario Internacional de Educación Ambiental (1975), donde se promulgan los principios rectores de la EA, reconociendo la educación como escenario que fomenta la generación de cambios en cuanto a conocimientos, actitudes y valores, para facilitar la posibilidad de asumir y responder ante los problemas ambientales desde todos los ámbitos propios del ser humano: ecológicos, económicos, tecnológicos, sociales, legislativos, culturales, éticos y estéticos; con el propósito de generar una conciencia que permita el análisis crítico de los conflictos, el reconocimiento de la responsabilidad frente a sus acciones y el compromiso necesario, mediado por la participación, para resolver, desde el nivel local, los retos de una sana convivencia con el medio.

Posteriormente, para 1977, Colombia participa con un delegado del Instituto Colombiano de Fomento de la Educación Superior, en la Conferencia Intergubernamental sobre Educación Ambiental, realizada en Tiblisi y, como resultado, surge el Programa Internacional de Educación Ambiental, además de un acuerdo en el que el país se compromete, junto a todas las naciones, a incluir a la EA en sus planes políticos, coincidiendo entonces con lo planteado durante la creación de la ya mencionada Comisión Asesora para la Educación Ecológica.
Durante la conferencia de Tiblisi se definieron los objetivos y logros básicos de la EA y, al tiempo, se adoptó el modelo pedagógico propuesto por la escuela activa, debido a que, por el alto grado de complejidad implícito en el análisis y gestión de las problemáticas ambientales, éstas requieren de un enfoque interdisciplinario que promueva la investigación y la reflexión crítica mientras, a su vez, permite la acción, con el propósito de impulsar al individuo a desempeñar una función productiva que mejore la calidad de vida y proteja el medio ambiente.

Ligado a ello, la Declaración de Tiblisi tiene en cuenta el concepto de desarrollo descrito en Cocoyoc, México, durante la Conferencia de Comercio y Desarrollo de las Naciones Unidas, durante la cual se reconoció que muchos de los problemas ambientales residen en las desigualdades presentes entre países desarrollados y subdesarrollados, hecho vinculado a los modelos "extraccionistas" predominantes desde la época colonial. Tales reflexiones llevaron a presentar respuestas frente a la necesidad de cambiar el modelo de desarrollo, cerrando la brecha entre los distintos países por medio del intercambio tecnológico y modelos sostenibles de crecimiento que aseguren la viabilidad de los ecosistemas a largo plazo, garantizando la cobertura de las necesidades básicas, como educación, salud y oportunidades (Zabala, 2008).

Lo anterior, representa uno de los primeros acercamientos al concepto contemporáneo de desarrollo sostenible, el cual se formuló diez años más tarde con el Informe Brundtland (1987), también conocido como "Nuestro futuro común"; el cual fue resultado del trabajo de la Comisión Mundial sobre el Medioambiente y Desarrollo, que se reunió por 
primera vez en 1983 y contó con la participación de Margarita Marino de Botero, gerente del Inderena en ese momento.

El Informe Brundtland hace un diagnóstico del lamentable estado del medio ambiente, respondiendo así a sucesos acontecidos durante los años ochenta, como los desastres de Bhopal y Chernóbil; frente a ello, plantea la necesidad de una convergencia entre los intereses económicos de desarrollo y la sostenibilidad, para que, de esta forma, sea posible dar fin al ritmo desenfrenado de destrucción, recurriendo a la utilización de tecnologías más eficientes y a la eliminación de las desigualdades entre primer y tercer mundo, de tal manera que se asegure el bienestar de las generaciones actuales sin comprometer a las generaciones futuras.

El desarrollo conceptual del Informe Brundtland llevó a incluir el desarrollo sostenible como nuevo enfoque central, para la EA, en el Congreso Internacional sobre la Educación y Formación relativa al Medio Ambiente (Moscú 1987), que comenzó por un diagnóstico del progreso en la implementación de la EA a nivel mundial, revelando una gran deficiencia institucional frente a lo propuesto durante la Conferencia de Tiblisi en 1977; por ello, se consideró adecuado impulsar una mayor inversión de recursos en proyectos de cooperación internacional, misiones asesoras, reuniones, encuentros y diversas publicaciones que partieran de una base regional (Orellana y Fauteux, 2002).

Luego de veinte años de la Cumbre de la Tierra, realizada en Estocolmo, se convoca a la Segunda Cumbre, esta vez en Río durante 1992; allí se reconoció la incapacidad general para realizar acciones efectivas frente a la protección del medio ambiente, por lo cual se planteó enfocar los esfuerzos hacia la realización de planes para el desarrollo sostenible mundial, siguiendo las definiciones propuestas en el Informe Brundtland. De acuerdo con ello, se plantearon tres acuerdos y dos instrumentos que contaban con fuerza de ley para los firmantes, entre ellos, la Agenda 21; un plan detallado de acciones que deben ser realizadas por todas las entidades gubernamentales en su campo de acción regional, atendiendo cada situación donde se presente un impacto humano en el medio ambiente.

El plan se enfocó en el objetivo de lograr un desarrollo sostenible, teniendo en cuenta la necesidad de cambiar los hábitos de consumo, erradicar la pobreza, fomentar la salud humana, conservar y gestionar los recursos y fortalecer el papel de los grupos principales, entre ellos, infancia y juventud, poblaciones indígenas y campesinas, mujeres, sindicatos, etc. La Agenda 21 reconoce, en su Capítulo 36, que el fomento de la educación, la capacitación y la toma de conciencia son medios de ejecución críticos para enfrentar las cuestiones ambientales y de desarrollo (Naciones Unidas, 1992).

La participación de Colombia, y su ratificación de los tratados formulados en estas conferencias, fueron de vital importancia en los cambios legislativos que tuvieron lugar durante la década del noventa, principalmente a partir de la Carta Magna de 1991, llamada "La Constitución Verde" por incluir más de 30 artículos que contemplaban la protección y defensa del medio ambiente, desde la forma de obligaciones para las autoridades públicas y privadas, entendiendo el medio ambiente como un derecho y un deber colectivo, y enmarcando el modelo económico dentro del concepto de desarrollo sostenible (Hernández, 2011). 
Finalmente, más cerca del presente siglo, la Ley 99 de 1993 crea el Ministerio de Ambiente y el Sistema Nacional Ambiental (SINA), mecanismos encargados de adoptar, junto con el Ministerio de Educación, los planes, programas docentes y el pensum a desarrollar en los distintos niveles de la educación nacional relacionados con el medio ambiente y los recursos naturales renovables; además de encargarse de la promoción, junto con dicho Ministerio, de los programas de divulgación y educación no formal, y de reglamentar la prestación del servicio ambiental.

Lo anterior se ratifica en 1994 con la Ley 115 o "Ley General de Educación”, que establece a las Ciencias Naturales y la Educación Ambiental como las primeras áreas obligatorias y fundamentales para la educación formal; mientras que, en conformidad con el Artículo 67 de la Constitución Política, se afirma que uno de los fines de la educación es, justamente:

La adquisición de una conciencia para la conservación, protección y mejoramiento del medio ambiente, de la calidad de la vida, del uso racional de los recursos naturales, de la prevención de desastres, dentro de una cultura ecológica y del riesgo y la defensa del patrimonio cultural de la Nación (MEN, 1994).

En esta ocasión, se reitera la importancia de generar, adquirir, promover y tomar conciencia frente a la relación con el medio ambiente, planteándola como uno de los principales objetivos de la educación, específicamente, desplegando una batería normativa destinada a cubrir lo concerniente a la materia, como punto ineludible para lograr una for- mación integral del individuo, al abarcar todas las esferas de la existencia e interacción humanas.

\section{Conclusiones}

Es posible deducir las condiciones históricas que permitieron el surgimiento de la Educación Ambiental, como formación discursiva dentro de la escuela en Colombia, a partir de las relaciones establecidas entre diferentes sectores de la sociedad, nacionales e internacionales, cada uno con sus propias perspectivas e intenciones económicas, políticas y sociales; sus directrices coincidieron en la necesidad de formar nuevos ciudadanos que incluyeran las preocupaciones ambientales como parte de su conciencia y, por consiguiente, de sus comportamientos y actitudes, respondiendo, desde la práctica, a su responsabilidad para con la comunidad, motivados por la promesa del mejoramiento en su calidad de vida.

Por otro lado, se destaca el concepto de sostenibilidad, que funcionó como integrador de las visiones conservacionistas y productivas, desde una perspectiva cientificista que le permitió establecerse como orientador de los esfuerzos de desarrollo en los distintos países, al tiempo que se presenta como método y objetivo por alcanzar. Finalmente, junto a ello, vale la pena resaltar la importancia que se le atribuiría a la escuela, como dispositivo que permitiría al discurso ambiental permear a los individuos $\mathrm{y}$ formar un régimen de verdad.

La parte arqueológica de la investigación partió de un seguimiento en las superficies del discurso, con el objeto de conformar el archivo de fuentes primarias para un período que comprende, desde el inicio de los años setenta, hasta finales de los noventa, 
considerado debido a los hitos destacados durante un rastreo histórico preliminar. Sin embargo, el desarrollo del estudio dio indicios que llevaron a plantear un seguimiento del discurso que comprendiera períodos anteriores, llegando incluso a la consulta de documentos de la época colonial y, en ámbitos exteriores, a la escuela, ejercicio que posibilitó un buen acercamiento a su procedencia.

A partir de la construcción del archivo fue posible observar cómo, mediante las relaciones entre diferentes fuerzas, y a través de las prácticas, tecnologías, objetos y sujetos configurados alrededor del discurso, y su llegada a la escuela, surge propiamente lo conocido como Educación Ambiental que, por consiguiente, permitió la formulación de su genealogía en esta investigación. 


\section{Referencias}

Acosta, A. (1977). Ciencias naturales integradas. Fichas de trabajo, manual de teoría, evaluaciones y práctica. Bogotá: Stella Editores.

Banco Internacional de Reconstrucción y Fomento. (1950). The basis of a development program for Colombia. Baltimore: John Hopkins University Press.

Barra, E. L. (2010). 50 años de la declaración del primer parque nacional natural. Revista La Barra. Obtenido el 31 de diciembre de 2019 desde https://www.revistalabarra.com/50-anos-de-ladeclaracion-del-primer-parque-nacional-natural/

Bastidas, J. M. (2013). De Rerum Natura, hitos para otra historia de la educación ambiental. Madrid: Bubok Publishing.

BirdLife International. (2020). Species factsheet: Grallaricula cucullata. Obtenido el 21 de octubre de 2020 desde http://www.birdlife.org

Black, R. (2012-Junio 4). Stockholm: Birth of the green generation. BBC News. Obtenido el 27 de enero de 2020 desde https://www.bbc.com/ news/science-environment-18315205

Botero, M. (1983). Ecodesarrollo, el pensamiento del decenio. Bogotá: Inderena.

Brundtland, G. H. (1987). Informe Brundtland. Oxford: Oxford University Press.
Carson, R. (1965). La primavera silenciosa. Madrid: Crítica.

Castro, E. (2004). El vocabulario de Michel Foucault. Un recorrido alfabético por sus temas, conceptos y autores. Bernal: Universidad Nacional de Quilmes.

Comitee on Foreign Affairs. (1971). Amending further the Peace Corps Act. Washington: US Government printing office.

Delworth, T. L. (2005). Oceanic forcing of the late 20th century Sahel drought. Geophysical Research Letters, Vol. 32. DOI: https://doi. org/10.1029/2005GL023316

Díaz, M. S. (1985). Compositor, Libreto de Ciencias Naturales. Bogotá: Acción Cultura Popular - Escuela Radiofónica.

Díaz, S. (2009). La Real Expedición Botánica. Credencial Historia, Vol. 240.

Dirección Nacional de Planeación. (1991). Una política ambiental para Colombia. CONPES No. 2544. Bogotá: DEPAC.

ECOFONDO. (2000-Mayo). Grupos de trabajo de Asamblea General de ECOFONDO. Bogotá: ECOFONDO.

Editorial Voluntad. (1976). Manual para las Ciencias Naturales de Voluntad: Metodologías, actividades y cuestionarios. Bogotá: Editorial Voluntad. 
Ehrlich, P. (1968). La bomba demográfica. Nueva York: Ballantine Books.

Ekelund. R. B., y Hébert, R. F. (1960). Historia de la teoría económica y su método. México: McGrawHill.

Escobar, A. (1986). La invención del desarrollo en Colombia. Lecturas de Economía, No. 20, pp. 9-35.

Fanlo, I. G. (2011). ¿Qué es un dispositivo? Foucault, Deleuze, Agamben. A parte rei, Vol. 74.

Foucault, M. (1992). Nietzsche, la genealogía, la historia. Valencia: Pretextos.

Foucault, M. (2002). Vigilar y castigar. Buenos Aires: Siglo. XXI.

Foucault, M. (2010). Arqueología del saber. Buenos Aires: Siglo XXI.

Gandhi, M. (1972). Man and Environment. Trabajo presentado en la Sesión Plenaria de las Naciones Unidas, Conference on Human Environment. Stockholm.

Gobernación de Antioquia. (2005). Lineamientos de políticas sobre uso y manejo mesurado de plaguicidas. Medellín: Gobernación de Antioquia.

Hernández, G. G. (2011). 20 años de la constitución verde. Vanguardia. Obtenido desde https://www. vanguardia.com/opinion/columnistas/gustavogalvis-hernandez/20-anos-de-la-constitucionverde-CCVL108569
Herrera, S. (1977). Ciencias naturales: Descubramos el mundo. Bogotá: Norma.

Hunsaker, D. (1972). National Parks in Colombia. Oryx, Vol. 11, No. 6. DOI: https://doi.org/10.1017/ S0030605300010711

Inderena. (1985). Campaña verde. El patrimonio común. El Tiempo. Bogotá.

Jácome, E. G. (1978). El oro en Colombia. Boletín de la Sociedad Geográfica de Colombia, Vol. 33, No. 113.

JSTOR. (S.f.). Lehmann Valencia, Federico Carlos (1914-1974). JSTOR, Global Plants. Obtenido el 5 de mayo de 2020 desde https://plants.jstor.org/ stable/10.5555/al.ap.person.bm000029840

Kragelund, E. (1992-Mayo 31). Deuda por naturaleza, un fracaso. El Tiempo. Obtenido desde https://www.eltiempo.com/archivo/documento/ MAM-126838

Lenis, Y. R. (2013). La historia de las áreas protegidas en Colombia, sus firmas de gobierno y las alternativas para la gobernanza. Trabajo presentado en el XXIX Congreso Latinoamericano de Sociología, Santiago de Chile.

Londoño, B. (1968). La Naturaleza: Introducción a las ciencias naturales. Bogotá: Editorial Voluntad.

MacDonald, L. I. (2003). IUCN: A history of constraint. Permanent workshop of the Centre for Philosophy of Law Higher Institute for Philosophy of the Catholic 
University of Louvain (UCL). Louvain: Catholic University of Louvain (UCL).

Martínez Boom, A. (2003). Currículo y modernización. Bogotá: Editorial Magisterio.

Meadows, D. H., Meadows, D. L., Randers, J., y Behrens, W. (1972). Los límites del crecimiento: informe al Club de Roma sobre el predicamento de la Humanidad. México: Fondo de Cultura Económica.

Megank, R. (1975). Colombia's national parks: An analysis of management, problems and perceived values. Corvallis: Oregon State University.

Ministerio de Educación Nacional (MEN). (1988a). Marco General. Ciencias Naturales y Salud: Propuesta programa curricular - sexto grado de educación básica. Bogotá: MEN.

Ministerio de Educación Nacional (MEN). (1988b). Marco general de ciencias naturales y salud: propuesta de programa curricular. Bogotá: MEN.

Ministerio de Educación Nacional (MEN). (1995). Lineamientos generales para una política nacional de educación ambiental. Bogotá: MEN.

Ministerio de Educación (MEN). (1994). Ley 115. Ley General de Educación. Bogotá: MEN.

Ministerio de Educación Nacional (MEN). (1998). Lineamientos Curriculares para Ciencias Naturales y Educación Ambiental. Bogotá: MEN.
Muñoz, A. S. (2017). La sociedad de control: una mirada a la educación del Siglo XXI desde Foucault. Revista de filosofía, Vol. 73. DOI: https:// doi.org/10.4067/S0718-43602017000100317

Naciones Unidas. (1972). Decisiones relativas a los informes de las comisiones. Informe de la Conferencia de las Naciones Unidas sobre el Medio Humano. Nueva York: Naciones Unidas.

Naciones Unidas. (1973). Informe de la Conferencia de las Naciones Unidas sobre el Medio Humano. Estocolmo, 5 a 16 de junio de 1972. Nueva York: Naciones Unidas. Obtenido desde https://www. dipublico.org/conferencias/mediohumano/ACONF.48-14-REV.1.pdf

Naciones Unidas. (1992). Agenda 21, Río de Janeiro, Brasil. Río: Naciones Unidas.

Orellana, I., y Fauteux, S. (2002). La educación ambiental a través de los grandes momentos de su historia. En Sauvé, L., Orellana, I., y Sato, M. (Eds.), Textos escogidos en educación ambiental. De una América a otra, Tomo I, pp. 39-54. Montreal: Les Publications ERE-UQAM.

Presidencia de la República. (1963). Decreto, número 1710 de 1963. Diario Oficial, No. 31169.

Prendergast, D., y Adams, W. (2003). Colonial wildlife conservation and the origins of the Society for the Preservation of the Wild Fauna of the Empire (1903-1914). Oryx, Vol. 37, No. 2. 
Purcell, F. (2014). Connecting realities: Peace Corps Volunteers in South America and the global war on poverty during the 1960s. Historia Crítica, No. 53. DOI: https://doi.org/10.7440/ histcrit53.2014.06

RCN. Radio. (2012-Noviembre 1). Ríos del Bajo Cauca antioqueño, los más contaminados de Colombia por mercurio. Obtenido el 17 de diciembre de 2019 desde https://www.rcnradio.com/colombia/riosdel-bajo-cauca-antioqueno-los-mas-contaminadosde-colombia-por-mercurio-30553

Redacción El Tiempo. (1994-Enero 23). La Venenosa Herencia del DDT. El Tiempo. Obtenido desde https://www.eltiempo.com/archivo/documento/ MAM-21822

Regodón, I. A. (2009). El hombre, la Tierra y el medio ambiente. Extremadura: Consejería de Educación.

República de Colombia. (1940). Decreto 1383 de 1940. Bogotá: República de Colombia.

República de Colombia. (1959). Artículo 13, Ley 2 de 1959. Bogotá: República de Colombia.

República de Colombia. (1974). Código Nacional de Recursos Naturales Renovables y de Protección al Medio Ambiente, Decreto 2811, 1974. Bogotá: República de Colombia.

Rousseau, J. J. (1762). Emilio o de la Educación. La Haya: El Aleph.
Salazar, B. (S.f.). El café como motor del desarrollo logístico a comienzos del Siglo XX. Logística en Colombia. Obtenido el 30 de diciembre de 2019 desde https://logisticaencolombia. jimdofree.com/historia-de-la-log\%C3\%ADstica-en -colombia/el-caf\%C3\%A9-motor-del-desarrollo$\log \% \mathrm{C} 3 \%$ ADstico-colombiano/

Sánchez, Á. (1991-Julio 7).Ecología: una constitución muy verde. El Tiempo. Bogotá.

Sánchez, A. (2017). Aproximación al concepto de Genealogía en Nietzsche y Foucault. La Torre del Virrey, No. 22.

Stapp, W. (1981). UNESCO's efforts in environmental education. EPA Journal - Education and Environment, Vol. 7, No. 1, pp. 8-11.

Susaeta Ediciones. (1983). Naturaleza y sociedad: Ciencias Naturales, nivel básico primario. Bogotá: Susaeta Ediciones.

Tobasura I. (2003). El movimiento ambiental colombiano, una aproximación a su historia reciente. Debates Ambientales, Vol. 26, pp. 107119.

Tobasura, I. (2011). El legado ecológico de "El libertador". Luna Azul, No. 32.

Triana, A. (2020). Escuelas normal rural, agropecuaria y campesinas en Colombia: 19341974. Revista Historia de la Educación Colombiana, Vol. 13, No. 13, pp. 201-230. 
UICN. (1980). World conservation strategy: Living resource conservation for sustainable development. Gland: IUCN-UNEP-WWF. DOI: https://doi. org/10.2305/IUCN.CH.1980.9.en

UNESCO. (1981). Proyecto principal de educación para América Latina y el Caribe. Quito: UNESCO.

UNESCO. (1984). Activities of the UNESCO-UNEP. International Environmental Education Program, 1975-1983. París: UNESCO.

UNESCO. (1985-Enero). Sexto Boletín. Proyecto principal de educación en América Latina y el Caribe. Santiago: OREALC, p. 12.

Urquidi, V. (2007). Desarrollo sustentable y cambio global. México: El Colegio de México. DOI: https:// doi.org/10.2307/j.ctvhn09kv.47

Valencia, L. E. (2009). Historia, realidad, pensamiento $y$ perspectivas de la acción comunal en Colombia. Corporación viva la ciudadanía. Obtenido desde http://viva.org.co/cajavirtual/svc0168/ art iculo0006.pdf

Vargas, H. (1993). Eco-cartilla del medio ambiente: guía metodológica. Bogotá: Funlibre, Inderena, Ministerio de Agricultura.

Vásquez, F. (2018-Febrero 5). Nechí, del río de oro al río de lodo. Las Dos Orillas. Obtenido el 17 de diciembre de 2019 desde https://www.las2orillas. co/nechi-del-rio-de-oro-al-rio-de-lodo/
Villaveces, J. (2019-Junio 26). Progreso y restauración: 200 años de la ley de Tierras. Portafolio. Obtenido desde https://www.portafolio .co/economia/liberalis mo-y-progresoconservatismo-y-restauracion-200-anos-de-la-leyde-tierras-530998

Zabala, I. (2008). Historia de la Educación Ambiental desde su discusión y análisis en los congresos internacionales. Revista de Investigación Instituto Pedagógico de Caracas, No. 63. 